\title{
An Beamforming Algorithm on Limited Feedback MIMO two-Way Relay Cognitive Networks
}

\author{
Jianwei Zhang and Guizhi Wang \\ Department of Computer Application, Henan University of Animal Husbandry and \\ Economy (Zhong zhou 450044, China) \\ 549006448@qq.com,522829031@qq.com
}

\begin{abstract}
In this paper, we study many antenna beamforming algorithm research in cognitive radio two-way relay system. In this system, two primary users exchange information by second users, and the second users to configure multiple antennas, a user through the superposition signal sends it to the primary users, therefore in this article research the key problem is how to divide the primary user information of the signal power and power of user information signal; How to design the main users of beamforming matrix; How to design the beam forming matrix of users. This paper uses the maximum system capacity optimization guidelines, even is a convex optimization problem, this paper adopts positive semidefinite optimization and second-order cone optimization, closed solution is obtained. The simulation results show that this method due to previous beam forming algorithm.
\end{abstract}

Keywords: cognitive radio two-way relay maximum system capacity beamforming

\section{Introduction}

In wireless communication, most of the limited radio spectrum resources assigned to the authorized users; However, authorized users in time, place, space and so on a series of dimensions not make full use of valuable spectrum, so the scholar put forward the Cognitive Radio, Cognitive Radio, CR) technology [1]. Cognitive radio technology basically is the use of authorized users in authorized spectrum without dimension transmission unauthorized user information. This technology is widely from proposed academic attention, become a research hotspot [2-4]. In cognitive radio, the first step is the most important step is the spectrum detection, spectrum detection accurate or not is not only related to cognitive radio users transmission, but also determines the amount of interference to primary users. This article is the study of cognitive radio spectrum detection problem. Many of the existing literature has proved that a single user cannot be reliable detection spectrum is being used, especially the main signal sent by a user is weak or when building hides under the condition of primary users. And relay technology can effectively solve the problem of weak signal due to shade, in relay technology transmission scheme, with two-way relay protocol transmission efficiency highest, confidentiality and superior performance, became a hot issue of research, but research collaboration in the two-way relay transmission protocol that results of spectrum sensing technology is rarely, at the same time can play more than the antenna signal is strong, easy to processing advantages, from a practical consideration will certainly increase the detection accuracy, therefore, in this paper, we study the two-way relay system for multiple antenna cognitive radio network. 
In the traditional study, cooperative spectrum sensing strategy usually introduce some users, finally will each inspection data or conclusions to the fusion center to make decisions [5]. Then a single antenna relay side to increase the detection accuracy, usually referred to as cognitive relay. In order to use the spectrum effectively, increase the spectrum efficiency, multi-antenna technology and two-way relay technology successively used in the article, it will inevitably bring some interference, the interference which can cause low detection efficiency, is the most effective ways for beam forming technology, using transceiver joint technology, eliminate interference suppression or, at the same time can through the beam forming technology makes information fusion, promote efficiency of detection.

System studied in the text, the primary users of relay nodes in the network using the second user can send both sides of the gap with time awareness of network base station information of user interaction. In spectrum perception of optimization criteria and user base station signal to noise ratio as an optimization goal, design the optimal beam forming algorithm, at the same time in the guarantee quality of service of primary user system, maximize user system signal-to-noise ratio. Outlined in this article is a nonconvex optimization function problem, which is positive definite planning. In this paper, the non convex problem into a convex optimization problem, can be closed solution, the detection probability of the result of simulation shows that this method is superior to other methods.

\section{System Model}

System model of the proposed scheme is shown in Figure 1, the system includes a primary user transceiver, the number of the second users is $K$, Primary user need through second users for information exchange, among them there is no link between information. In this article, the second user has the effect of two aspects, first is the spectrum sensing and detecting primary users if they are using authorized spectrum; the second is as the primary users of relay, help the primary users to communicate. Hypothesis from the start, synchronization mechanism is perfect, each step is synchronization, and time the user USES the amplification forwarding trunking protocol for forwarding. Primary user configuration $N$ receiving antenna, at the same time, each second user $N$ antenna configuration, second user base station $M$ antenna configuration.

Two time slot as shown in Figure 1 is the direction of the signal transmission, in the time slot 1 , the main users of the sender and the base station sends a signal to the user at the same time a user receives a signal, will signal after superposition; In slot 2, users will be good signal broadcast to the primary user receiver and user base. At this time user base station can carry out information fusion to complete the main user is occupy the frequency in a sentence, improve the detection probability.

Which at the first time slot, user base station and primary user base station all users to send information to The Times; Second user time slot, will process after the signal is sent to the primary user and secondary user base station. The dotted line represents the interference to primary user. Do not break general, primary user base station for the user and user base station for the user to send information respectively, and assumes that the primary user base station and there is no direct communication link between primary user. This system is frequency division duplex system, in the first time slot, the user $i(i=1,2)$ sends a signal $\mathbf{S}_{i}$ to a second user, the second user receives the signal can be expressed as 


$$
\mathbf{x}_{R}=\sum_{i=1}^{2} \sqrt{P_{i}} \mathbf{h}_{i} \mathbf{s}_{i}+\mathbf{z}
$$

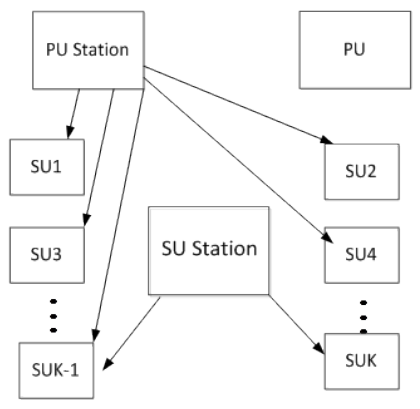

slot1

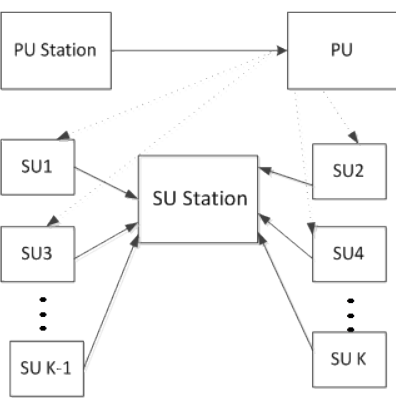

slot2

Figure 1. Cognitive Radio Two-way Relay System

Among them, $\mathbf{s}_{i} \in \square^{N \times 1}$ is the signal information for users $i$ to send, because the power normalization, so $E\left(\mathbf{S}_{i} \mathbf{S}_{i}^{\mathrm{B}}\right)=\mathbf{I}_{N} \cdot \square^{N \times M}$ denotes matrix element of the plural, and the matrix $N$ and $M$ of the ranks; $\mathbf{I}_{N}$ is on behalf of the $N \times N$ unit matrix; $E(\bullet)$ is on behalf of the averaging operation; $(\bullet)^{\mathbf{B}}$ is on behalf of the matrix conjugate gear operation; $\mathbf{h}_{i} \in \square^{\boldsymbol{M} \times \boldsymbol{N}}$ is the user to the relay node's channel matrix, obey Rayleigh fading channel. In the first time slot of sending power $\sqrt{P_{i}}$ to second user $i$. For relay terminal receiving noise matrix, this paper consider the additive white gaussian noise, the mean to 0 , noise variance can be expressed as $E\left(\mathbf{z z}^{\mathrm{H}}\right)=\sigma^{2} \mathbf{I}_{\boldsymbol{M}}$. In this paper, due to the estimate of the signal is not clear, so the power uses the average power allocation scheme. Assumes that the primary user base station and second user base all know channel state information.

In the second time slot, the second user will receive the signal is signal processing, the beamforming treatment to the primary user base and second user base, so as to transmit the primary user information and second user base stations spectrum detection purposes. Which $\mathbf{T}_{j}$ represents the first time a signalsent by a user $j(j=1,2, \cdots, K)$, it can be written as:

$$
\mathbf{T}_{j}=\sum_{j=11}^{K} \sqrt{P_{j}} \mathbf{W} \mathbf{G}_{j} \mathbf{x}_{j}+\mathbf{v}_{j}
$$

Which is the primary user base station or second user base station additive white gaussian noise, the mean to 0 , the variance for $\sigma^{2} ; \mathbf{G}_{j}$ is the second user to the primary user channel, In order to ensure that the user's normal communication, user interference to primary user cannot exceed a specific value, represented here

$$
\sum_{i=1}^{K}\left\|\mathbf{G}_{i}\right\|^{2} P_{i} \leq \eta
$$

(1) primary user receiver to receive signals

At the second time slot, and two receiving end receives the signal $\boldsymbol{y}_{\mathbf{1}}$ and $\boldsymbol{y}_{\mathbf{2}}$, respectively, which were represented as 


$$
\begin{aligned}
& y_{1}=\mathbf{h}_{1}^{\mathrm{H}} \mathbf{W} \mathbf{x}+n_{1} \\
& y_{2}=\mathbf{h}_{2}^{\mathrm{H}} \mathbf{W} \mathbf{x}+n_{2}
\end{aligned}
$$

Which $n_{k}$ is the noise at the receiving $k$ terminal, the same subject to additive white gaussian noise, the mean to 0 , noise variance can be expressed as $E\left(n_{k} n_{k}^{*}\right)=\sigma^{2}$. According to the matrix of equivalent change, can be obtained:

$$
\begin{aligned}
& y_{1}=\sqrt{P_{1}} \mathbf{W}^{H} \mathbf{H}_{1} \mathbf{h}_{1} \mathbf{s}_{1}+\sqrt{P_{2}} \mathbf{W}^{H} \mathbf{H}_{1} \mathbf{h}_{2} \mathbf{s}_{2}+\mathbf{W}^{H} \mathbf{H}_{1} \mathbf{z}+n_{1} \\
& y_{2}=\sqrt{P_{1}} \mathbf{W}^{H} \mathbf{H}_{2} \mathbf{h}_{1} \mathbf{s}_{1}+\sqrt{P_{2}} \mathbf{W}^{H} \mathbf{H}_{2} \mathbf{h}_{2} \mathbf{s}_{2}+\mathbf{W}^{H} \mathbf{H}_{2} \mathbf{z}+n_{2}
\end{aligned}
$$

Among them $\mathbf{H}_{k}=\operatorname{diag}\left(\mathbf{h}_{k}\right)$, when $k=1,2$. And $\mathbf{W}=\operatorname{diag}\left\{\mathbf{W}^{\mathrm{H}}\right\}$, which $\operatorname{diag}\{\bullet\}$ represents the diagonalization. And beamforming matrix $\mathbf{W}$ from the optimization solution, and through the control channel is transmitted to the user. And for users of 1 , to know their information $\mathbf{s}_{1}$ know so in the main user base station, so the formula (6) the first $\sqrt{P_{1}} \mathbf{W}^{H} \mathbf{H}_{1} \mathbf{h}_{1} \mathbf{s}_{1}$ is known. As a result, the signal $\mathbf{s}_{2}$ can be directly need to know. Similarly, in formula (7) the second is known, so the signal $\mathbf{s}_{1}$ can be need to know. Can define the remaining signals $y_{1}^{\prime}=y_{1}-\sqrt{P_{1}} \mathbf{W}^{H} \mathbf{H}_{1} \mathbf{h}_{1} \mathbf{s}_{1}$ and $y_{2}^{\prime}=y_{2}-\sqrt{P_{2}} \mathbf{W}^{H} \mathbf{H}_{2} \mathbf{h}_{2} \mathbf{s}_{2}$ can be as judgment, can get the signal $\mathbf{s}_{1}$ an $\mathbf{s}_{2} \mathrm{~d}$ the need.

(2)second user base station receives the signal

In this system model, assuming that the cognitive network does not consider any interference to primary user network, therefore, the main user application two-way relay network communication, user base station for opportunistic transmission through induction channel, in the second time slot, user base station receives the signal can be represented as:

$$
y_{3}=\mathbf{H}_{3} \mathbf{W}^{H} \mathbf{x}+n_{3}
$$

Among them, $\mathbf{H}_{3}=\operatorname{diag}\left(\mathbf{h}_{3}\right)$ is a user to the channel matrix between user base station. According to matrix knowledge into (9), type (9) can be written as

$$
y_{3}=\sqrt{P_{1}} \mathbf{W}^{H} \mathbf{H}_{3} \mathbf{h}_{1} \mathbf{s}_{1}+\sqrt{P_{2}} \mathbf{W}^{H} \mathbf{H}_{3} \mathbf{h}_{2} \mathbf{s}_{2}+\mathbf{W}^{H} \mathbf{H}_{3} \mathbf{z}+n_{3}
$$

In time user base station receives the signal from the main user receives a signal use is different, in the main user receiving signal is to get each other's information, and in time the user receiving signal is for spectrum detection, the focus of this article is to time spectrum detection aspects of the user base station.

\section{Feedback Scheme}

Due to channel vector matrix, so this article will study the feedback mechanism of $\mathbf{h}_{i}$ elements need to be sure both obey the plural (independent identically distributed gaussian distribution, the mean zero, variance of 1 . The feedback of this study is based on the codes, on the basis of the fixed number of so called limited feedback, limited feedback user preset time and user base, every time all determine the code in this 
collection. Set code this collection for $C$, including $2^{B}$ element, and each element $\square^{N \times 1}$ vector, so it can be used $\left(\mathbf{c}_{1}, \ldots, \mathbf{c}_{2^{B}}\right)$, which is each second user feedback to the user B number of bits in the base station. Based on the literature [6] quantization scheme, this scheme adopts the random code, by literature [7] can know, random code this quantification can achieve optimal system performance. In the option code standards, this article adopts the way of string distance, choose the shortest distance, the specific criteria, such as type (11)

$$
\hat{\mathbf{H}}_{i}=\underset{\mathbf{W} \in \mathrm{C}}{\arg \min } \sin (\theta)
$$

Among them $\theta$ representative the Angle between $\mathbf{h}_{i}$ and $\mathbf{c}$ the various elements, and $\sin (\theta)$ represents the chordal distance of system, in order to solve the above problem, in order to facilitate analysis, this article uses the literature [8] decomposition approach:

$$
\tilde{\mathbf{h}}_{i}=\hat{\mathbf{h}}_{i}+\tilde{\mathbf{h}}_{i} \sin \theta
$$

Among them $\tilde{\mathbf{h}}_{i}=\mathbf{h}_{i} /\left\|\mathbf{h}_{i}\right\|, \theta_{i}$ is on behalf of the Angle between the channels in the channel direction and the direction, according to its statistical properties, according to the literature [13]

where

$$
\begin{gathered}
E\left(\sin ^{2} \theta\right)=\frac{(N-1) \delta}{M} \\
\mathbf{H}=\mathbf{A} \hat{\mathbf{H}}+\mathbf{B G}
\end{gathered}
$$

$$
\begin{array}{r}
\mathbf{A}=\mathbf{D}(\mathbf{I}-\boldsymbol{\theta})^{1 / 2} \\
\mathbf{B}=\mathbf{D}(\boldsymbol{\theta})^{1 / 2} \\
\text { and } \mathbf{D}=\operatorname{diag}\left\{\left\|\mathbf{h}_{1}\right\|, \cdots\left\|\mathbf{h}_{K}\right\|\right\}, \boldsymbol{\theta}=\operatorname{diag}\left\{\sin ^{2} \theta_{1}, \cdots, \sin ^{2} \theta_{K}\right\} 。
\end{array}
$$

\section{Limited Feedback Spectrum Sensing}

This part adopts the spectrum sensing, the relatively small amount of calculation, and high efficiency of energy awareness technology, energy sensing technology is shown in Figure 2.

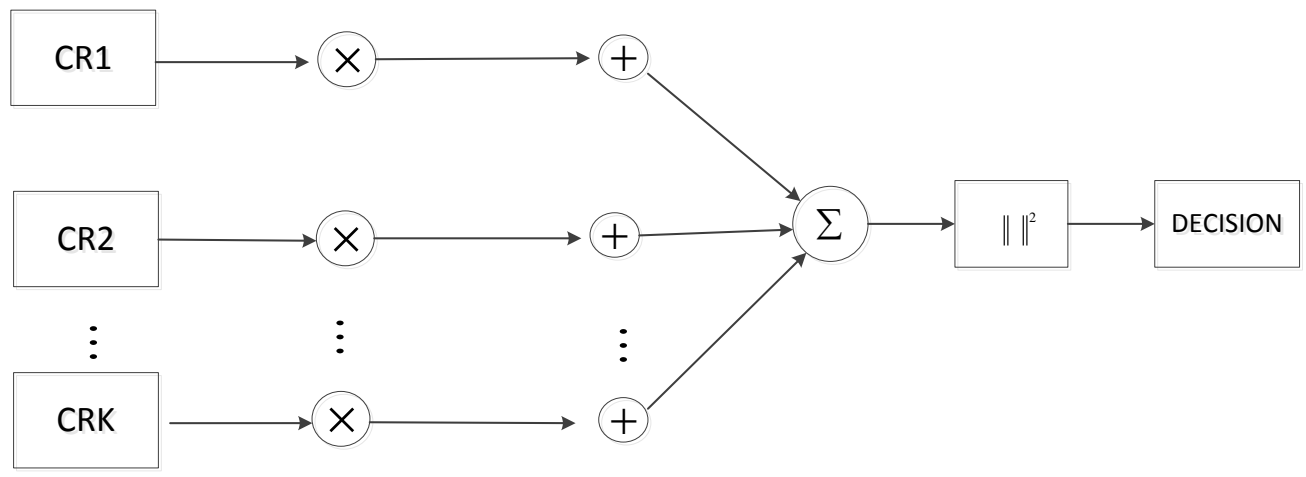

Figure 2. Energy Detection Scheme 
Energy sensing technology is seen as a binary hypothesis test problem of hypothesis testing problem, will be located in synchronization accurately in the system, the received signal can be represented as binary hypothesis

$$
y_{3}=\left\{\begin{array}{lc}
\mathbf{H}_{3} \mathbf{W}^{H} \mathbf{x}+n_{3} & H_{0} \\
\sqrt{P_{1}} \mathbf{W}^{H} \mathbf{H}_{3} \mathbf{h}_{1} \mathbf{s}_{1}+\sqrt{P_{2}} \mathbf{W}^{H} \mathbf{H}_{3} \mathbf{h}_{2} \mathbf{s}_{2}+\mathbf{W}^{H} \mathbf{H}_{3} \mathbf{z}+n_{3} & H_{1}
\end{array}\right.
$$

Among them, $H_{0}$ and $H_{1}$ said the primary user signal respectively not occupied and occupied.

Assumptions $n_{3}$ and $\mathbf{z}$ are complex additive white gaussian noise channel, and its distribution $c N\left(0, \sigma^{2}\right)$ and $c N\left(0, \sigma^{2} \mathbf{I}_{N}\right)$. In addition $\mathbf{s}_{1}$ and $\mathbf{s}_{2}$ as zero-mean gaussian random variables, the variance of 1 . According to random matrix knowledge, can get is a gaussian random distribution, the distribution can be expressed as type (18),

$$
y_{3} \square\left\{\begin{array}{cc}
c N\left(0, \sigma_{1}^{2}\right) & H_{0} \\
c N\left(0, \sigma_{2}^{2}\right) & H_{1}
\end{array}\right.
$$

And $\sigma_{1}^{2}$ and $\sigma_{2}^{2}$ according to the random matrix can be obtained:

$$
\sigma_{1}^{2}=\sigma^{2}\left(\mathbf{w}^{\mathrm{H}} \mathbf{H}_{3} \mathbf{H}_{3}^{\mathrm{H}} \mathbf{w}+1\right)
$$

and

$$
\sigma_{2}^{2}=P_{1} \mathbf{w}^{\mathrm{H}} \mathbf{H}_{3} \mathbf{h}_{1} \mathbf{h}_{1}^{\mathrm{H}} \mathbf{w}+P_{2} \mathbf{w}^{\mathrm{H}} \mathbf{H}_{3} \mathbf{h}_{2} \mathbf{h}_{2}^{\mathrm{H}} \mathbf{w}+\sigma^{2}\left(\mathbf{w}^{\mathrm{H}} \mathbf{H}_{3} \mathbf{H}_{3}^{\mathrm{H}} \mathbf{w}+1\right)
$$

Superposition of the energy through user base station can be obtained:

$$
S=\sum_{i=1}^{N}\left|y_{3}\right|^{2}
$$

Due to the superposition, and $S$ obeys degrees of freedom for $\mathrm{N}$ chi-square distributed variable, depending on the central limit theorem and the chi-square statistical features, you can get the following statistics, i.e

$$
S \square\left\{\begin{array}{l}
N\left(N \sigma_{1}^{2}, 2 N \sigma_{1}^{4}\right), H_{0} \\
N\left(N \sigma_{2}^{2}, 2 N \sigma_{2}^{4}\right), H_{1}
\end{array}\right.
$$

According to maximum likelihood detection, can get the decision threshold for $\lambda$, according to the inspection can be the detection probability $P_{D}$ and false alarm probability $P_{F}$, as shown below

$$
P_{D}=\operatorname{Pr}\left(S>\lambda \mid H_{1}\right)=Q\left(\frac{\lambda-N \sigma_{2}^{2}}{\sqrt{2 N \sigma_{2}^{4}}}\right)
$$




$$
P_{F}=\operatorname{Pr}\left(S>\lambda \mid H_{0}\right)=Q\left(\frac{\lambda-N \sigma_{1}^{2}}{\sqrt{2 N \sigma_{1}^{4}}}\right)
$$

$Q(\square)$ as a standard gaussian distribution of cumulative distribution function, through the reasonable design, can get the corresponding false-alarm probability and detection probability,

$$
\lambda=\sqrt{2 N \sigma_{1}^{4}} Q^{-1}\left(P_{F}\right)+N \sigma_{1}^{2}
$$

To bring (24) to (25), can get the relationship between the detection probability and false alarm probability

$$
P_{D}=Q\left(\frac{\sqrt{2 N \sigma_{1}^{4}} Q^{-1}\left(P_{F}\right)+N \sigma_{1}^{2}-N \sigma_{2}^{2}}{\sqrt{2 N \sigma_{2}^{4}}}\right)
$$

Defines the received signal-to-noise ratio

$$
S N R=\frac{P_{1} \mathbf{w}^{\mathrm{H}} \mathbf{H}_{3} \mathbf{h}_{1} \mathbf{h}_{1}^{\mathrm{H}} \mathbf{w}+P_{2} \mathbf{w}^{\mathrm{H}} \mathbf{H}_{3} \mathbf{h}_{2} \mathbf{h}_{2}^{\mathrm{H}} \mathbf{w}}{\sigma^{2}\left(\mathbf{w}^{\mathrm{H}} \mathbf{H}_{3} \mathbf{H}_{3}^{\mathrm{H}} \mathbf{w}+1\right)}
$$

Will type (27) into type (26) and so can be two-way relay detection probability of cognitive system are as follows:

$$
P_{D}=Q\left(\frac{\sqrt{2 N} Q^{-1}\left(P_{F}\right)+N S N R}{\sqrt{2 N}(1+S N R)}\right)
$$

As can be seen from the type (28), the detection probability is a function of the SNR increases, so to maximize the signal to noise ratio, can effectively improve the detection probability of the system.

\section{Under the Condition of Limited Feedback Spectrum Perception Optimization}

According to the analysis of part 4, based on the condition of limited feedback optimal spectrum perception function, so as to improve the performance of spectrum sensing. Its main means is to minimize the signal-to-noise ratio, therefore, must first determine the optimization function of this paper, the optimization problem can be expressed as

$$
\begin{array}{ll}
\underset{W}{\operatorname{MAX}} & S N R \\
\text { s.t. } & \sigma_{1}^{2} \leq \varphi_{1} \\
& \sigma_{2}^{2} \leq \varphi_{2} \\
& S \leq P_{\text {MAX }}
\end{array}
$$

$\varphi_{1}$ and $\varphi_{2}$ are tolerance of interference noise, respectively, $P_{\text {MAX }}$ to the maximum power of the entire network, in a certain system, these values are known, and therefore, in here, can be as a constant. And under the condition of limited feedback, the feedback 
channel is no longer a full channel state information, so the SNR and other involved and the channel state information are calculated according to the feedback of the estimate, i. e.,

$$
\begin{gathered}
\sigma_{1}^{2}=\sigma^{2}\left(\mathbf{w}^{\mathrm{H}} \hat{\mathbf{H}}_{3} \hat{\mathbf{H}}_{3}^{\mathrm{H}} \mathbf{w}+1\right) \\
\sigma_{2}^{2}=P_{1} \mathbf{w}^{\mathrm{H}} \hat{\mathbf{H}}_{3} \hat{\mathbf{h}}_{1} \hat{\mathbf{h}}_{1}^{\mathrm{H}} \mathbf{w}+P_{2} \mathbf{w}^{\mathrm{H}} \hat{\mathbf{H}}_{3} \hat{\mathbf{h}}_{2} \hat{\mathbf{h}}_{2}^{\mathrm{H}} \mathbf{w}+\sigma^{2}\left(\mathbf{w}^{\mathrm{H}} \hat{\mathbf{H}}_{3} \hat{\mathbf{H}}_{3}^{\mathrm{H}} \mathbf{w}+1\right) \\
S N R=\frac{P_{1} \mathbf{w}^{\mathrm{H}} \hat{\mathbf{H}}_{3} \hat{\mathbf{h}}_{1} \hat{\mathbf{h}}_{1}^{\mathrm{H}} \mathbf{w}+P_{2} \mathbf{w}^{\mathrm{H}} \hat{\mathbf{H}}_{3} \hat{\mathbf{h}}_{2} \hat{\mathbf{h}}_{2}^{\mathrm{H}} \mathbf{w}}{\sigma^{2}\left(\mathbf{w}^{\mathrm{H}} \hat{\mathbf{H}}_{3} \hat{\mathbf{H}}_{3}^{\mathrm{H}} \mathbf{w}+1\right)}
\end{gathered}
$$

Due to the type (30) is a convex optimization function, so the need to convert it to optimize function, namely the definition $\mathbf{W}=\mathbf{w} \mathbf{w}^{H}$, the optimization problem can be converted into

$$
\begin{array}{ll}
\text { find } \mathbf{W} \\
\text { s.t. } & \operatorname{Tr}\left[\left(\hat{\mathbf{H}}_{3}+\hat{\mathbf{h}}_{1}-\sigma^{2} \hat{\mathbf{h}}_{2}\right) \mathbf{W}\right] \geq \varphi_{1} t \\
& \operatorname{Tr}\left[\left(\hat{\mathbf{H}}_{3}-\sigma^{2} \hat{\mathbf{h}}_{1}\right) \mathbf{W}\right] \geq \varphi_{2} t \\
& S \leq P_{M A X}
\end{array}
$$

According to equation (33) can see, only to find $t$ in a reasonable scope, to find the optimal value $t_{\max }$, the value $t$ in the range of $\left[t_{l}, t_{u}\right]$, find the optimal value $t_{\max }$, the paper adopts the search point method, specific steps are as follows:

Step 1. Set the reasonable initial value $t_{l}$ and $t_{u}$;

Step 2. The setup $t=\left(t_{l}+t_{u}\right) / 2$ and solution (33);

Step 3. If the type (33), $t_{l}$ is set to $t$; Otherwise, $t_{u}$ set to $t$;

Step 4, if $t_{u}-t_{l}<\delta$ to step 5. Otherwise, starting from the fourth step;

Step 5 is the main feature vector for the optimal $\mathbf{W}$.

\section{Simulation}

In this part, using the computer simulation to show the advanced nature of the proposed scheme; In order to simplify the simulation, this paper argues that the user is equal to twoway relay distance. Do not break general, RS and RS is the user to the main user distance to the user from two times and three times. In this simulation, $M=8, N=2, K=8$, User and set a time equal to the maximum power of the relay sends, i.e., $P_{R}=P_{k}=P_{t}$. In the simulation, set up different stages of boundaries are the primary user interference. In limited feedback mechanism, the simulation uses the stochastic high 'flow pattern quantitative code, the number of iterations for 8 times. In order to compare the feedback scheme under the 
condition of superiority, this paper simulates the feedback bits for the 6 bit and 12 bit. In order to compare convenience,

Figure 3 for the missed detection probability under the change of SNR change trend, as can be seen from the curve, with the increase of SNR, can see the missed detection probability. It just conforms to the formula (32) analysis.

Figure 4 is the number of missed detection probability in the SU change curve, and its change trend, with the increase of SU, the missed detection probability gradually decline. Is also reflected in the type (33).

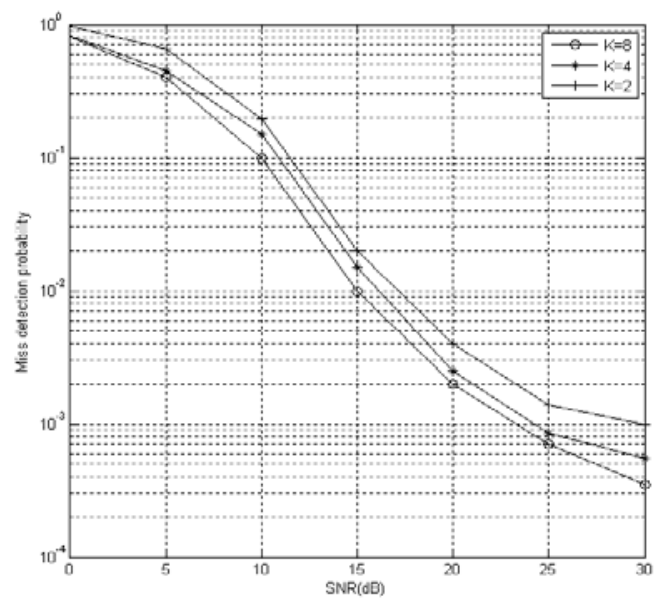

\section{Figure 3. The Curve of the Missed Detection Probability vs SNR}

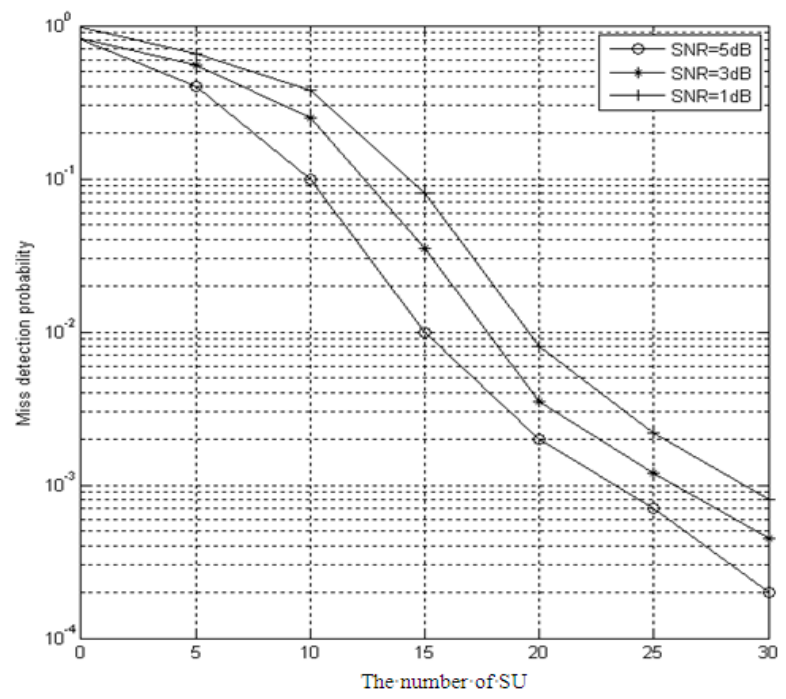

Figure 4. Missed Detection Probability with the SU

\section{Conclusion}

Based on two-way relay in the cognitive system is studied based on an energy sensing bea forming algorithm, this algorithm through the simulation shows the effectiveness of the proposed method, and it can be seen that the solution is optimal solution. 


\section{References}

[1] V. Chakravarthy, X. Li and R. L. Zhou, “A Novel Hybrid Overlay/Underlay Cognitive Radio Waveform in Frequency Selective Fading Channels”, Proc. 4th International Conference on Cognitive Radio Oriented Wireless Networks and Communications, (2009), pp. 1-6.

[2] V. Chakravarthy, Z. Q. Wu and M. Temple, “Cognitive Radio Centric Overlay/Underlay Waveform”, Proc. 3rd IEEE Symposium on New Frontiers in Dynamic Spectrum Access Networks, (2008), pp. 1-10.

[3] V. Chakravarthy, Z. Q Wu and A. Shaw, "A General Overlay/Underlay Analytic Expression Representing Cognitive Radio Waveform”, Proc. International Waveform Diversity and Design Conference, (2007), pp. 69-73.

[4] F. F. Digham, M. S. Alouini and M. K. Simon, “On the Energy Detection of Unknown Signals over Fading Channels”, Proc. IEEE International Conference on Communications (ICC’03), (2003) May, pp. 3575-3579.

[5] A. Ghasemi and E. S. Sousa, "Collaborative Spectrum Sensing for Opportunistic Access in Fading Environments”, First IEEE International Symposium on New Frontiers in Dynamic Spectrum Access Networks (DySPAN 2005), (2005), pp. 131-136.

[6] S. P. Chen, W. B. Wang and X. Zhang, "Performance Analysis of Multiuser Diversity in Cooperative MultiRelay Networks under Rayleigh-Fading Channels”, IEEE Trans. on Wireless Commun., vol. 8, no. 8, (2009), pp. 3415-3419.

[7] J. Oh and W. Choi, "A Hybrid Cognitive Radio System: A Combination of Underlay and Overlay Approaches”, IEEE Vehicular Technology Conference Fall (VTC 2010-Fall), (2010), pp. 1-5.

[8] S. Sriram, S. Vishwanath and S. Jafar, "On the Capacity of Cognitive Relay Assisted Gaussian Interference Channel”, proc. of 2008 International Symposium on Information Theory, (2008) July, pp. 1-5.

[9] J. Chen, S. Li and S. Deng, "Route Selection Strategy Combined with Spectrum Characteristic in Cognitive Radio Networks”, IJSEIA, vol. 2, no. 2, (2008) April, pp. 119-128.

[10] C. Song, K. Kim, W. Park and J. Kim, "Performance Analysis of Signal feature detection using Spectral Correlation Function for Cognitive Radio Environments”, IJMUE, vol. 1, no. 3, (2006) September, pp. 32-36.

[11] J. Lee, "Blind Spectrum Sensing Techniques for Cognitive Radio System”, IJMUE, vol. 3, no. 2, (2008) April, pp. 103-116.

[12] T. (Tom) Oh, Y. B. Choi, M. Guthrie, K. Harold, D. Copeland and T.-H. Kim, "Reeling In Cognitive Radio: The Issues of Regulations and Policies Affecting Spectrum Management”, IJFGCN, vol. 3, no. 3, (2010) September, pp. 71-79.

\section{Authors}

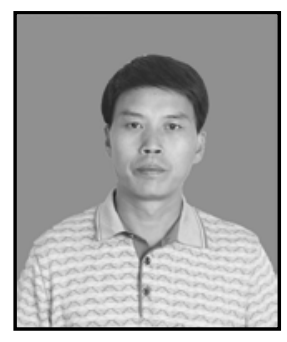

Jianwei Zhang, received the Bachelor's degree in Electric Technology and Master's degree in Computer Software and Theory from Zhengzhou University, China in 1994 and 2005 respectively. He is an associate professor of Henan University of Animal Husbandry and Economy. His research interests on Semantic Recognition and Wireless Communication.

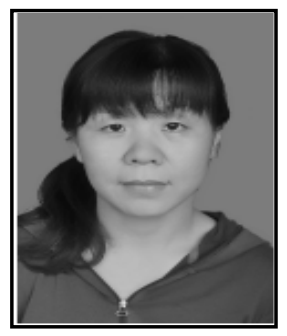

Guizhi Wang, received the B.Eng degree in Computer Science and Technology from Henan Normal University and the M.Eng degree in Computer Software and Theor from Zhengzhou University, CHINA in 1995 and 2008 respectively. She is an associate professor and deputy director of the Department of Computer Applications, Henan University of Animal Husbandry and Economy. Her current research interests on Data Mining and Wireless Communication. 